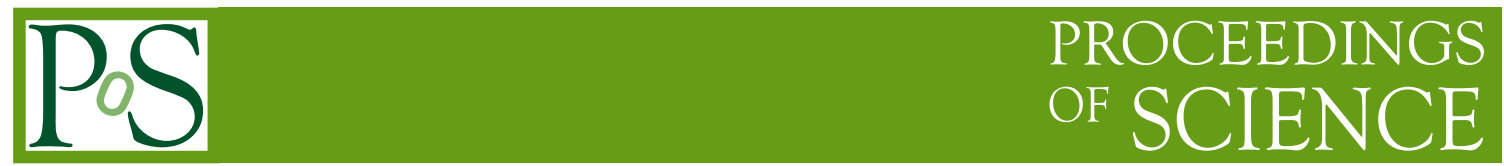

\title{
Hexagons, Heptagons and Octogons in $\mathrm{N}=4$ SYM
}

\author{
Claude Duhr*t \\ Institut für theoretische Physik, ETH Zürich, \\ Wolfgang-Paulistr. 27, CH-8093, Switzerland \\ E-mail: duhrceitp.phys.ethz.ch
}

We report on the progress in the computation of multi-loop multi-leg scattering amplitudes in the planar $\mathscr{N}=4$ Super Yang-Mills theory. We review analytic results for scattering amplitudes and Wilson loops in this theory, as well as new mathematical tools for the computation of Feynman integrals introduced in this context.

"Loops and Legs in Quantum Field Theory" 11th DESY Workshop on Elementary Particle Physics April 15-20, 2012

Wernigerode, Germany

\footnotetext{
* Speaker.

†This work was supported by the ERC grant "IterQCD". We would like to thank the organizers for their invitation to the conference and the participants for the many stimulating discussions.
} 


\section{Introduction}

In recent years there has been tremendous progress in the analytic computation of multi-loop multi-leg color-ordered scattering amplitudes in the planar $\mathscr{N}=4$ Super Yang-Mills (SYM) theory. At the core of this progress lies a duality which relates scattering amplitudes to the vacuum expectation value of certain Wilson loops computed along a lightlike polygonal contour constructed out of the momenta entering the amplitude. While this duality was originally discovered at strong coupling [1], it was soon realized that a similar duality relates Wilson loops and maximally-helicityviolating (MHV) amplitudes at weak coupling [2, 3, 4, 5, 6, 7, 8]. Recently, a supersymmetric generalization of Wilson loops was proposed which allows to extend the duality beyond the MHV helicity configurations $[9,10]$.

The Wilson loops possess a conformal symmetry, which manifests itself at the level of the scattering amplitudes as a dual conformal symmetry that is independent from the usual conformal symmetry of $\mathscr{N}=4 \mathrm{SYM}$. It was shown that by combining the generators of the ordinary and dual conformal symmetries, one obtains the algebra of an infinite-dimensional Yangian symmetry [11]. Yangian symmetries are known to arise in the context of integrable systems, thus increasing the hope that scattering amplitudes and Wilson loops in planar $\mathscr{N}=4$ SYM can be solved exactly for any value of the coupling, reconciling in this way the strong and weak coupling computations.

The Yangian symmetry is however broken by infrared divergences, and the (bosonic) Wilson loops satisfy an anomalous conformal Ward identity [5]. The Ward identity allows one to express the Wilson loop, and thus the dual MHV amplitude, at any loop order as an iteration of the oneloop Wilson loop, augmented by a finite function of conformal cross ratios, the so-called remainder function. While the remainder function is known to vanish for four and five-point amplitudes, it is in general non-zero and its functional form cannot be obtained from the conformal Ward identities alone.

The aim of this contribution is to review the recent analytic results for scattering amplitudes and Wilson loops at weak coupling in planar $\mathscr{N}=4$ SYM. One of the cornerstones used in the derivation of these results are a collection of new mathematical tools to deal in an efficient way with the algebraic and combinatorial properties of multiple polylogarithms. The outline of this contribution is as follows: In Section 2 we review mathematical tools that were used to obtain new results for scattering amplitudes and Wilson loops in planar $\mathscr{N}=4 \mathrm{SYM}$. In Section 3 we summarize the available results for scattering amplitudes in general kinematics, before turning to recent results in restricted kinematics for high numbers of loops and / or external legs in Sections 4 and 5.

\section{Multiple polylogarithms and symbols}

It is well known that large classes of Feynman integrals can be expressed in terms of multiple polylogarithms and generalizations thereof. In this section we give a brief account on multiple polylogarithms and discuss some of their mathematical properties. In particular, we review the symbol map, a linear map that associates to every multiple polylogarithm a certain tensor which captures the essential combinatorics underlying the functional equations among polylogarithmic functions. 
Multiple polylogarithms are a generalization of the ordinary logarithm $\ln z$ and the classical polylogarithms $\operatorname{Li}_{n}(z)$. They are defined through the iterated integral $[12,13]$

$$
G\left(a_{1}, \ldots, a_{n} ; z\right)=\int_{0}^{z} \frac{d t}{t-a_{1}} G\left(a_{2}, \ldots, a_{n} ; t\right),
$$

with $G(z)=1$ and where $a_{i}, z \in \mathbb{C}$. In the special case where all the $a_{i}$ 's are zero, we define, using the obvious vector notation $\vec{a}_{n}=(\underbrace{a, \ldots, a}_{n})$,

$$
G\left(\overrightarrow{0}_{n} ; z\right)=\frac{1}{n !} \ln ^{n} z
$$

The number $n$ of elements $a_{i}$, counted with multiplicities, is called the weight of the multiple polylogarithm.

Iterated integrals form a shuffle algebra, i.e., it is possible to express the product of two multiple polylogarithms of weight $n_{1}$ and $n_{2}$ as a linear combination with integer coefficients of multiple polylogarithms of weight $n_{1}+n_{2}$,

$$
G\left(a_{1}, \ldots, a_{n_{1}} ; z\right) G\left(a_{n_{1}+1}, \ldots, a_{n_{1}+n_{2}} ; z\right)=\sum_{\sigma \in \Sigma\left(n_{1}, n_{2}\right)} G\left(a_{\sigma(1)}, \ldots, a_{\sigma\left(n_{1}+n_{2}\right)} ; z\right),
$$

where $\Sigma\left(n_{1}, n_{2}\right)$ denotes the set of all shuffles of $n_{1}+n_{2}$ elements, i.e., the subset of the symmetric group $S_{n_{1}+n_{2}}$ defined by

$$
\Sigma\left(n_{1}, n_{2}\right)=\left\{\sigma \in S_{n_{1}+n_{2}} \mid \sigma^{-1}(1)<\ldots<\sigma^{-1}\left(n_{1}\right) \text { and } \sigma^{-1}\left(n_{1}+1\right)<\ldots<\sigma^{-1}\left(n_{1}+n_{2}\right)\right\} .
$$

A way to deal with the various functional equations satisfied by multiple polylogarithms is given by the symbol map, a linear map which associates to every multiple polylogarithm an element in the tensor algebra over the group of rational functions. Various (equivalent) definitions have been given in the literature for the symbol of a multiple polylogarithm $[14,15,16,17,18,19]$. One possible way to define the symbol of a multiple polylogarithm is to consider its total differential [13],

$$
d G\left(a_{n-1}, \ldots, a_{1} ; a_{n}\right)=\sum_{i=1}^{n-1} G\left(a_{n-1}, \ldots, \hat{a}_{i}, \ldots, a_{1} ; a_{n}\right) d \ln \left(\frac{a_{i}-a_{i+1}}{a_{i}-a_{i-1}}\right),
$$

and to define the symbol recursively by [17]

$$
\mathscr{S}\left(G\left(a_{n-1}, \ldots, a_{1} ; a_{n}\right)\right)=\sum_{i=1}^{n-1} \mathscr{S}\left(G\left(a_{n-1}, \ldots, \hat{a}_{i}, \ldots, a_{1} ; a_{n}\right)\right) \otimes\left(\frac{a_{i}-a_{i+1}}{a_{i}-a_{i-1}}\right) .
$$

As an example, the symbols of the classical polylogarithms and the ordinary logarithms are given by

$$
\mathscr{S}\left(\operatorname{Li}_{n}(z)\right)=-(1-z) \otimes \underbrace{z \otimes \ldots \otimes z}_{(n-1) \text { times }} \text { and } \mathscr{S}\left(\frac{1}{n !} \ln ^{n} z\right)=\underbrace{z \otimes \ldots \otimes z}_{n \text { times }} .
$$

In addition the symbol satisfies the following identities,

$$
\begin{aligned}
& \ldots \otimes(a \cdot b) \otimes \ldots=\ldots \otimes a \otimes \ldots+\ldots \otimes b \otimes \ldots, \\
& \ldots \otimes( \pm 1) \otimes \ldots=0 .
\end{aligned}
$$


While in special cases multiple polylogarithms can be expressed through classical polylogarithms and ordinary logarithms only, there are no such simple formulae known in general. It is known however that up to weight three all multiple polylogarithms can be expressed in terms of classical polylogarithms and ordinary logarithms only. The first time an irreducible multiple polylogarithms appears is thus at weight four, and there is a necessary and sufficient criterion (at least conjecturally) that allows one to determine whether a given combination of multiple polylogarithms of weight four can be expressed in terms of classical polylogarithms only. Indeed, if we define a linear operator $\delta$ acting on tensors of rank four by

$$
\delta(a \otimes b \otimes c \otimes d)=(a \wedge b) \wedge(c \wedge d),
$$

with $a \wedge b=a \otimes b-b \otimes a$, then it follows from the conjecture of ref. [20] that a combination $f$ of multiple polylogarithms of weight four can be expressed through classical polylogarithms only if and only if the symbol of $f$ satisfies

$$
\delta[\mathscr{S}(f)]=0 .
$$

In the next sections we will see that the algebraic concepts introduced in this section provide an ideal language to discuss the structure of scattering amplitudes in planar $\mathscr{N}=4 \mathrm{SYM}$.

\section{3. $\mathscr{N}=4$ SYM scattering amplitudes in general kinematics}

In this section we review the available analytic results for scattering amplitudes and Wilson loops in planar $\mathscr{N}=4 \mathrm{SYM}$. It follows from the dual conformal Ward identities satisfied by the Wilson loops that the remainder function for the four and five-point amplitudes vanish, and thus these amplitudes are obtained to all loop orders by iterating the corresponding one-loop amplitudes. It then follows that the first non-trivial MHV remainder function arises for the two-loop six-point amplitude. The six-point MHV remainder function is a function of the three conformal cross ratios

$$
u_{1}=\frac{x_{13}^{2} x_{46}^{2}}{x_{36}^{2} x_{41}^{2}}, \quad u_{2}=\frac{x_{15}^{2} x_{24}^{2}}{x_{14}^{2} x_{25}^{2}}, \quad u_{3}=\frac{x_{26}^{2} x_{35}^{2}}{x_{25}^{2} x_{36}^{2}},
$$

with $x_{i j}^{2}=\left(x_{i}-x_{j}\right)^{2}$ and $p_{i}=x_{i}-x_{i+1}$. The corresponding Wilson loop diagrams were evaluated analytically in refs. [21, 22] by exploiting the Regge exactness of (the logarithm of) the Wilson loop. The results were expressed in terms of a complicated combination of several thousand multiple polylogarithms of weight four. In ref. [17] the symbol map was applied for the first time in physics and it was shown that the symbol of the two-loop six-point MHV remainder function satisfies eq. (2.10). As a consequence, it is possible to simplify the results of refs. [21, 22] and to rewrite them in a form which only involves classical polylogarithms [17],

$$
\begin{aligned}
R_{6}^{(2)}\left(u_{1}, u_{2}, u_{3}\right)= & \sum_{i=1}^{3}\left(L_{4}\left(x_{i}^{+}, x_{i}^{-}\right)-\frac{1}{2} \operatorname{Li}_{4}\left(1-1 / u_{i}\right)\right)-\frac{1}{8}\left(\sum_{i=1}^{3} \operatorname{Li}_{2}\left(1-1 / u_{i}\right)\right)^{2} \\
& +\frac{1}{24} J^{4}+\frac{\pi^{2}}{12} J^{2}+\frac{\pi^{4}}{72}
\end{aligned}
$$

with $x_{i}^{ \pm}=u_{i} x^{ \pm}$and

$$
x^{ \pm}=\frac{u_{1}+u_{2}+u_{3}-1 \pm \sqrt{\Delta}}{2 u_{1} u_{2} u_{3}}
$$


where $\Delta=\left(1-u_{1}-u_{2}-u_{3}\right)^{2}-4 u_{1} u_{2} u_{3}$. Furthermore, the functions appearing in Eq. (3.2) are defined by

$$
L_{4}\left(x^{+}, x^{-}\right)=\sum_{k=0}^{3} \frac{(-1)^{k}}{(2 k) ! !} \ln ^{k}\left(x^{+} x^{-}\right)\left(\ell_{4-k}\left(x^{+}\right)+\ell_{4-k}\left(x^{-}\right)\right)+\frac{1}{8 ! !} \ln ^{4}\left(x^{+} x^{-}\right),
$$

with

$$
\ell_{n}(x)=\frac{1}{2}\left[\operatorname{Li}_{n}(x)-(-1)^{n} \operatorname{Li}_{n}(1 / x)\right] \text { and } J=\sum_{i=1}^{3}\left(\ell_{1}\left(x_{i}^{+}\right)+\ell_{1}\left(x_{i}^{-}\right)\right) .
$$

Finally, it was shown in ref. [17] that the square roots in Eq. (3.3) can be interpreted as cross ratios in momentum twistor space, e.g.,

$$
x_{1}^{+}=-\frac{\langle 3456\rangle\langle 1245\rangle}{\langle 1456\rangle\langle 2345\rangle} .
$$

Momentum twistors were introduced by Hodges in ref. [23] and are four-component objects $Z_{i}$ living in a three-dimensional complex projective space. They provide a way to encode the kinematics of a massless scattering, the kinematic invariants being related to the determinants formed out of four twistors,

$$
x_{i j}^{2} \sim\langle(i-1) i(j-1) i\rangle,
$$

with

$$
\langle i j k l\rangle=\operatorname{det}\left(Z_{i} Z_{j} Z_{k} Z_{l}\right)=\left(\begin{array}{cccc}
Z_{i}^{1} & Z_{j}^{1} & Z_{k}^{1} & Z_{l}^{1} \\
Z_{i}^{2} & Z_{j}^{2} & Z_{k}^{2} & Z_{l}^{2} \\
Z_{i}^{3} & Z_{j}^{3} & Z_{k}^{3} & Z_{l}^{3} \\
Z_{i}^{4} & Z_{j}^{4} & Z_{k}^{4} & Z_{l}^{4}
\end{array}\right) .
$$

Up to now, the two-loop six-point remainder function is the only case for which a fully analytic expression is known. There was, however, progress in determining the symbols of scattering amplitudes at two loops and beyond. In particular, in ref. [24] the anomalous dual superconformal symmetry was used to determine the symbols of all two-loop MHV remainder remainder functions. It can then be checked that for more than six external legs, the symbol of the MHV remainder function does no longer satisfy the condition (2.10), i.e., MHV remainder functions for more than six external legs cannot be expressed in terms of classical polylogarithms only. Despite the fact that the symbols of all two-loop MHV remainder functions have been known for more than a year now, no function is known that matches these symbols beyond six points.

Furthermore, progress was made in determining the structure of scattering amplitudes beyond two loops. In ref. [25] the symbol of the three-loop six-point MHV remainder function was determined by making some reasonable assumptions on the arguments that can appear in the symbol of this function, and it was shown that the most general symbol of this type consistent with collinear and Regge limits and the collinear operator product expansion [26] depends on only two free parameters. The results of ref. [25] were shortly after confirmed in ref. [27] and the two free parameters were fixed by using the superconformal anomaly equation. The technique of ref. [25] was recently also applied to the two-loop six-point MHV amplitude in ref. [28] where its symbol was determined. In this case it was possible to find a one-fold integral representation of the amplitude with the same symbol. 
While there was a lot of progress in determining the symbols of scattering amplitudes in planar $\mathscr{N}=4 \mathrm{SYM}$, no fully analytic result at the function level is known beyond the two-loop six-point MHV amplitude. It is however possible to obtain analytic results for scattering amplitudes with more loops and legs by considering amplitudes in specific kinematic limits. These results will be reviewed in the next sections.

\section{Scattering amplitudes in two-dimensional kinematics}

In ref. [29] Alday and Maldacena considered scattering amplitudes in planar $\mathscr{N}=4 \mathrm{SYM}$ at strong coupling in the limit where all external momenta lie in a common two-dimensional plane. The two-dimensional kinematics require the amplitude to depend on an even number of external momenta, and the remainder function of the simplest non-trivial MHV amplitude, the six-point remainder function, approaches a constant. It then follows that the first non-trivial MHV scattering amplitude in two dimensions is the two-loop eight-point MHV amplitude, which was evaluated analytically at weak coupling in ref. [30]. The result of ref. [30] takes a strikingly simple form and is expressed as a product of four logarithms. Shortly after it was shown using numerical analysis that this simple structure is present in all two-loop MHV remainder functions in two dimensions [31],

$$
R_{n}^{(2)}=-\frac{1}{2} \sum_{\left(i_{1}, \ldots, i_{8}\right) \in S} \ln u_{i_{1} i_{5}} \ln u_{i_{2} i_{6}} \ln u_{i_{3} i_{7}} \ln u_{i_{4} i_{8}}-\frac{\pi^{4}}{72}(n-4),
$$

where $u_{i j}$ denote dual conformal cross ratios and the sum extends over the set

$$
S=\left\{\left(i_{1}, \ldots, i_{8}\right): 1 \leq i_{1}<\ldots<i_{8} \leq n \text { and } i_{k}-i_{k+1} \text { odd }\right\} .
$$

For $n=8$, eq. (4.1) coincides with the result of ref. [30]. Recently, several conjectures have been made regarding the structure of remainder functions in two-dimensions with more loops [32, 33], but so far no analytic result beyond two loops is available.

\section{Scattering amplitudes in the multi-Regge limit}

Besides two-dimensional kinematics, there is another region of phase space for which analytic results for multi-loop multi-leg amplitudes can be obtained. While it is known that the MHV remainder functions vanish in the Euclidean region, where all invariants are negative, in multiRegge kinematics (MRK), where the total incoming energy is much larger than the momentum transfers in the $t$-channel $[34,35,36,37]$, there is a Minkowski region, relevant to $2 \rightarrow 4$ scattering, where the MHV remainder functions are non zero. In the case of the six-point MHV remainder function this Minkowski region can be reached by analytically continuing $R_{6}\left(u_{1}, u_{2}, u_{3}\right)$ according to $u_{1} \rightarrow e^{-2 \pi i}\left|u_{1}\right|$ and then taking the limit $u_{1} \rightarrow 1$ while keeping the ratios

$$
\frac{u_{2}}{1-u_{1}} \equiv \frac{1}{(1+w)\left(1+w^{*}\right)} \text { and } \frac{u_{3}}{1-u_{1}} \equiv \frac{w w^{*}}{(1+w)\left(1+w^{*}\right)}
$$

fixed [34]. In the MRK limit, the six-point remainder function can be written in the form

$$
\left.R_{6}\right|_{\mathrm{MRK}}=2 \pi i \sum_{\ell=2}^{\infty} \sum_{n=0}^{\ell-1} a^{\ell} \ln ^{n}\left(1-u_{1}\right)\left[g_{n}^{(\ell)}\left(w, w^{*}\right)+2 \pi i h_{n}^{(\ell)}\left(w, w^{*}\right)\right] .
$$


The six-point MHV remainder function was computed at two loops in leading-logarithmic accuracy (LLA) and next-to-leading-logarithmic accuracy (NLLA) [38, 39] and at three loops up to next-tonext-to-leading-logarithmic accuracy (NNLLA) [25]. Furthermore, all two-loop MHV remainder functions in LLA are known [40].

In ref. $[39,41]$ an all-loop integral formula for the six-point remainder function in MRK was proposed,

$$
\begin{aligned}
\left.e^{R_{6}+i \pi \delta}\right|_{\mathrm{MRK}} & =\cos \pi \omega_{a b} \\
& +i \frac{a}{2} \sum_{n=-\infty}^{\infty}(-1)^{n}\left(\frac{w}{w^{*}}\right)^{\frac{n}{2}} \int_{-\infty}^{+\infty} \frac{d v}{v^{2}+\frac{n^{2}}{4}}|w|^{2 i v} \Phi_{\operatorname{Reg}}(v, n)\left(-\frac{1}{\sqrt{u_{2} u_{3}}}\right)^{\omega(v, n)},
\end{aligned}
$$

where $a$ denotes the 't Hooft coupling and

$$
\omega_{a b}=\frac{1}{8} \gamma_{K}(a) \log |w|^{2} \text { and } \delta=\frac{1}{8} \gamma_{K}(a) \log \frac{|w|^{2}}{|1+w|^{4}},
$$

and $\gamma_{K}(a)$ is the cusp anomalous dimension, known to all orders [42]. The impact factor $\Phi_{\operatorname{Reg}}(v, n)$ and the BFKL eigenvalue $\omega(v, n)$ admit the perturbative expansion

$$
\omega(v, n)=-a\left(E_{v, n}+a E_{v, n}^{(1)}+\mathscr{O}\left(a^{2}\right)\right) \text { and } \Phi_{\operatorname{Reg}}(v, n)=1+a \Phi_{\operatorname{Reg}}^{(1)}(v, n)+\mathscr{O}\left(a^{2}\right)
$$

and are known up to NLLA [38, 41, 43].

In ref. [44] it was argued that the functions $g_{n}^{(\ell)}\left(w, w^{*}\right)$ and $h_{n}^{(\ell)}\left(w, w^{*}\right)$ can be expressed to all orders in perturbation theory in terms of the single-valued harmonic polylogarithms (SVHPLs) introduced by Brown [45]. It is then possible to write down a linear combination of SVHPLs of a given weight, and the coefficients of the linear combination can be fixed by requiring its Taylor expansion to match the series obtained by considering the integral in eq. (5.3) as a contour integral in the complex $v$ plane and summing up residues. In this way, the six-point MHV remainder function was determined up to ten loops in LLA and up to nine loops in NLLA [44]. Finally we conclude by mentioning that six-point MHV and NMHV remainder function in LLA in MRK are related by [46]

$$
R_{6, \mathrm{NMHV}}^{\mathrm{LLA}}=\int d w \frac{w^{*}}{w} \frac{\partial}{\partial w^{*}} R_{6, \mathrm{MHV}}^{\mathrm{LLA}} .
$$

As the MHV results are known up to ten loops, it is then trivial to obtain analytic results for $R_{6, \mathrm{NMHV}}^{\mathrm{LLA}}$ up to the same loop order [44].

\section{Conclusion}

In this contribution we have reported on the progress made in recent years in the computation of scattering amplitudes and Wilson loops in the planar $\mathscr{N}=4$ SYM theory. This progress was made possible by a deeper understanding of the algebraic and combinatorial structures underlying multiple polylogarithms, a class of special functions through which large classes of Feynman integrals can be expressed. In addition, this deeper understanding revealed that all the analytic results available in the literature for scattering amplitudes in planar $\mathscr{N}=4 \mathrm{SYM}$ are characterized by a remarkable and unexpected simplicity. 
So far it is not clear yet how much of the simplicity of the scattering amplitudes in $\mathscr{N}=4$ SYM will be present in amplitudes in more realistic theories like QCD. It was nonetheless already shown that, by using the new mathematical tools inspired by $\mathscr{N}=4$ SYM, the two-loop helicity amplitudes for a Higgs boson plus three gluons [47] can be rewritten in a much simpler form that involves only classical polylogarithms $[19,48]$. This gives hope that also scattering amplitudes in other theories have a hidden simplicity that waits yet to be uncovered.

\section{References}

[1] L. F. Alday and J. M. Maldacena, JHEP 0706 (2007) 064 [arXiv:0705.0303 [hep-th]].

[2] J. M. Drummond, G. P. Korchemsky and E. Sokatchev, Nucl. Phys. B 795 (2008) 385 [arXiv:0707.0243 [hep-th]].

[3] A. Brandhuber, P. Heslop and G. Travaglini, Nucl. Phys. B 794 (2008) 231 [arXiv:0707.1153 [hep-th]].

[4] J. M. Drummond, J. Henn, G. P. Korchemsky and E. Sokatchev, Nucl. Phys. B 795 (2008) 52 [arXiv:0709.2368 [hep-th]].

[5] J. M. Drummond, J. Henn, G. P. Korchemsky and E. Sokatchev, Nucl. Phys. B 826 (2010) 337 [arXiv:0712.1223 [hep-th]].

[6] J. M. Drummond, J. Henn, G. P. Korchemsky and E. Sokatchev, Nucl. Phys. B 815 (2009) 142 [arXiv:0803.1466 [hep-th]].

[7] Z. Bern, M. Czakon, D. A. Kosower, R. Roiban and V. A. Smirnov, Phys. Rev. Lett. 97 (2006) 181601 [arXiv:hep-th/0604074].

[8] Z. Bern, L. J. Dixon, D. A. Kosower, R. Roiban, M. Spradlin, C. Vergu and A. Volovich, Phys. Rev. D 78 (2008) 045007 [arXiv:0803.1465 [hep-th]].

[9] L. J. Mason and D. Skinner, JHEP 1012 (2010) 018 [arXiv:1009.2225 [hep-th]].

[10] S. Caron-Huot, JHEP 1107 (2011) 058 [arXiv:1010.1167 [hep-th]].

[11] J. M. Drummond, J. M. Henn and J. Plefka, JHEP 0905 (2009) 046 [arXiv:0902.2987 [hep-th]].

[12] A. B. Goncharov, Math. Research Letters, 5 (1998), 497-516 [arXiv:1105.2076].

[13] A. B. Goncharov, (2001) [math/0103059v4].

[14] A.B. Goncharov, [arXiv:0908.2238v3 [math.AG]].

[15] K. T. Chen, Bull. Amer. Math. Soc. 83 (1977) 831.

[16] F. Brown, Annales scientifiques de l'ENS 42, fascicule 3, 371 (2009) [math/0606419].

[17] A. B. Goncharov, M. Spradlin, C. Vergu and A. Volovich, Phys. Rev. Lett. 105 (2010) 151605 [arXiv:1006.5703 [hep-th]].

[18] C. Duhr, H. Gangl and J. R. Rhodes, [arXiv:1110.0458 [math-ph]].

[19] C. Duhr, JHEP 1208 (2012) 043 [arXiv:1203.0454 [hep-ph]].

[20] A. B. Goncharov, Proc. of the International Congress of Mathematicians, Vol. 1, 2 (Zurich, 1994), 374387, Birkhauser, Basel, 1995.

[21] V. Del Duca, C. Duhr and V. A. Smirnov, JHEP 1003 (2010) 099 [arXiv:0911.5332 [hep-ph]]. 
[22] V. Del Duca, C. Duhr and V. A. Smirnov, JHEP 1005 (2010) 084 [arXiv:1003.1702 [hep-th]].

[23] A. Hodges, arXiv:0905.1473 [hep-th].

[24] S. Caron-Huot, JHEP 1112 (2011) 066 [arXiv:1105.5606 [hep-th]].

[25] L. J. Dixon, J. M. Drummond and J. M. Henn, JHEP 1111 (2011) 023 [arXiv:1108.4461 [hep-th]].

[26] L. F. Alday, D. Gaiotto, J. Maldacena, A. Sever and P. Vieira, JHEP 1104 (2011) 088 [arXiv:1006.2788 [hep-th]].

[27] S. Caron-Huot and S. He, JHEP 1207 (2012) 174 [arXiv:1112.1060 [hep-th]].

[28] L. J. Dixon, J. M. Drummond and J. M. Henn, JHEP 1201 (2012) 024 [arXiv:1111.1704 [hep-th]].

[29] L. F. Alday and J. Maldacena, JHEP 0911 (2009) 082 [arXiv:0904.0663 [hep-th]].

[30] V. Del Duca, C. Duhr and V. A. Smirnov, JHEP 1009 (2010) 015 [arXiv:1006.4127 [hep-th]].

[31] P. Heslop and V. V. Khoze, JHEP 1011 (2010) 035 [arXiv:1007.1805 [hep-th]].

[32] P. Heslop and V. V. Khoze, JHEP 1111 (2011) 152 [arXiv:1109.0058 [hep-th]].

[33] T. Goddard, P. Heslop and V. V. Khoze, arXiv:1205.3448 [hep-th].

[34] J. Bartels, L. N. Lipatov and A. Sabio Vera, Phys. Rev. D 80 (2009) 045002 [arXiv:0802.2065 [hep-th]].

[35] R. C. Brower, H. Nastase, H. J. Schnitzer and C.-I. Tan, Nucl. Phys. B 814 (2009) 293 [arXiv:0801.3891 [hep-th]].

[36] R. C. Brower, H. Nastase, H. J. Schnitzer and C.-I. Tan, Nucl. Phys. B 822 (2009) 301 [arXiv:0809.1632 [hep-th]].

[37] V. Del Duca, C. Duhr and E. W. N. Glover, JHEP 0812 (2008) 097 [arXiv:0809.1822 [hep-th]].

[38] L. N. Lipatov and A. Prygarin, Phys. Rev. D 83 (2011) 045020 [arXiv:1008.1016 [hep-th]].

[39] L. N. Lipatov and A. Prygarin, Phys. Rev. D 83 (2011) 125001 [arXiv:1011.2673 [hep-th]].

[40] A. Prygarin, M. Spradlin, C. Vergu and A. Volovich, Phys. Rev. D 85 (2012) 085019 [arXiv:1112.6365 [hep-th]].

[41] V. S. Fadin and L. N. Lipatov, Phys. Lett. B 706 (2012) 470 [arXiv:1111.0782 [hep-th]].

[42] N. Beisert, B. Eden and M. Staudacher, J. Stat. Mech. 0701, P01021 (2007) [hep-th/0610251].

[43] J. Bartels, L. N. Lipatov and A. Sabio Vera, Eur. Phys. J. C 65 (2010) 587 [arXiv:0807.0894 [hep-th]].

[44] L. J. Dixon, C. Duhr and J. Pennington, arXiv:1207.0186 [hep-th].

[45] F. C. S. Brown, C. R. Acad. Sci. Paris, Ser. I 338 (2004).

[46] L. Lipatov, A. Prygarin and H. J. Schnitzer, arXiv:1205.0186 [hep-th].

[47] T. Gehrmann, M. Jaquier, E. W. N. Glover and A. Koukoutsakis, JHEP 1202 (2012) 056 [arXiv:1112.3554 [hep-ph]].

[48] A. Brandhuber, G. Travaglini and G. Yang, JHEP 1205 (2012) 082 [arXiv:1201.4170 [hep-th]]. 